\title{
基于非球面镜像差效应的长焦深高斯光束均匀化光学系统设计
}

\author{
杨 振, 郭金民, 张建隆, 于祥燕, 张 全 \\ (哈尔滨工业大学 航天学院光电子信息科学与技术系, 黑龙江 哈尔滨 150080)
}

\begin{abstract}
摘要: 近红外高斯激光束在强激光与材料相互作用、激光清洗、激光燃烧诊断等热点研究领域中发 挥着重要的作用。然而, 高斯光束能量分布的不均匀性阻碍了这些领域的深入发展。为提高工作效 率和测量精度, 实际应用中往往期望光束能量在较大工作距离内呈均匀分布, 但现有光束整形方法 无法同时满足长焦深和高激光耐受功率要求。为此, 本文基于非球面像差效应提出并设计了一种新 型长焦深高斯激光束均匀化光学系统, 系统由非球面光束均匀化系统和球面长焦准直系统两部分组 成, 所有透镜均采用熔融石英并在其表面镀有增透膜, 能够实现 $99.9 \%$ 的光学系统传输效率。系统 工作波段为 $1064 \mathrm{~nm}$, 工作距离为 $1000 \mathrm{~mm}$, 系统总长为 $135.2 \mathrm{~mm}$, 耐受激光功率不小于 $300 \mathrm{~W}$ 。 设计结果表明: 整形后的平顶高斯光束有效焦深为 $\pm 100 \mathrm{~mm}$, 光束均匀性 $\geqslant 95 \%$, 会聚角为 17.52 $\mathrm{mrad}$, 能够满足上述应用场景的实际需求。本文设计的光束整形系统相比于其他激光光束均匀化系 统, 具有结构简单、易于加工、成本低、焦深长、耐受激光功率高、光束均匀化效果好的特点。 关键词: 像差效应; 长焦深; 高斯光束; 光束均匀化系统; 耐受激光功率

中图分类号：O439 文献标识码：A 文章编号：1001-8891(2020)04-0320-08
\end{abstract}

\section{Optical System Design for Gaussian Beam Uniformization with Long Focal Depth Based on Aberration Effect of Aspheric Mirror}

\author{
YANG Zhen, GUO Xinmin, ZHANG Jianlong, YU Xiangyan, ZHANG Quan \\ (Department of Optoelectronic Information Science and Technology, School of Astronautics, Harbin Institute of Technology,
} Harbin 150080, China)

\begin{abstract}
Near infrared Gaussian laser beams are crucial in many research studies, such as the interaction between intense laser and materials, laser cleaning, and laser combustion diagnostics. However, the non-uniformity of energy distribution of Gaussian beams hinders the further development of these fields. To improve cleaning efficiency and measuring accuracy, beam energy is typically expected to distribute uniformly within a larger working distance in practical applications. Nevertheless, the current beam shaping methods cannot satisfy requirements of long focal depth and high laser power tolerance simultaneously. Therefore, a novel optical system of Gaussian beam uniformization with long focal depth is proposed and designed based on the aberration effect of aspheric surface lens, which comprises an aspheric beam uniformization system and a spherical long focal collimation system. All lenses are fabricated with fused quartz and coated with antireflective film on the surface, which can achieve a $99.9 \%$ transmission efficiency in the optical system. The working wavelength of the system is $1064 \mathrm{~nm}$, the working distance is $1000 \mathrm{~mm}$, the total length of the system is $135.2 \mathrm{~mm}$, and the laser power is higher than $300 \mathrm{~W}$. The design results show that the effective focal depth of the shaped flat-topped Gaussian beam is $\pm 100 \mathrm{~mm}$, the uniformity of the beam is better than $95 \%$, and the convergence angle is $17.52 \mathrm{mrad}$, which satisfy the requirements of the abovementioned application fields. Compared with other laser beam uniformization systems, the beam shaping system designed in this study possesses a simple structure and affords easy processing, low cost, long focal length, high laser power tolerance, and good beam
\end{abstract}


uniformization.

Key words: aberration effect, long focal depth, Gaussian beam, beam uniformization system, laser power tolerance

\section{0 引言}

强激光与材料相互作用 ${ }^{[1]}$ 、激光清洗 ${ }^{[2]}$ 、激光燃 烧诊断 ${ }^{[3]}$ 是近年来激光应用技术领域中的热点研究 领域。强激光与材料相互作用时不可避免会对材料 表面及内部产生损伤, 目前国际上通常采用损伤阈 值（即作用于材料表面能够引起损伤的平均激光功 率密度或能量密度) 来定量评价激光对材料破坏的 程度。然而, 激光器输出的光束一般为高斯或类高 斯分布光斑, 这种光斑能量分布具有中间高两边弱 的特点, 采用这种光束作用于材料本身, 将使得材 料与激光相互作用时空间各点的能量分布不均匀, 导致采用平均激光功率密度或平均能量密度表征激 光损伤阈值不具有统一的规范和标准。即便不同激 光器厂商的平均功率/能量密度相同, 但由于激光的 其他参数不同 (如激光脉宽、光束质量等), 激光峰 值功率/能量密度也常常不完全一致。因此, 通常采 用不同厂商的激光器进行损伤评价不具有可比性和 参考性, 这给建立统一的评价激光损伤标准带来了 极大困扰。

激光清洗被誉为 21 世纪的绿色清洗方式, 未来 有望应用于航空航天、高铁、海洋、核电等重要工 程领域 ${ }^{[4]}$ 。激光清洗相比于传统的化学清洗, 能够有 效降低对环境的破坏程度; 相比于人工清洗, 能够 极大提升清洗的效率。然而, 激光清洗目前多采用 振镜扫描方式进行清洗, 利用激光清洗头内部的摆 镜快速旋转, 使激光光斑逐点作用于被清洗工件的 表面, 完成清洗。这种方式缺点之一是其清洗时的 光斑分布不均匀, 导致清洗时光斑中心位置的效率 高, 光斑两侧低, 即清洗效率不够高。往往清洗一 次时, 光斑中心位置清洗的彻底, 两侧存在漏洗或 清除不净的情况。

激光燃烧诊断是研究航空发动机及燃气轮机等 大中型燃烧装置的有利手段。激光燃烧诊断能够通 过激光无接触式获得燃烧场内部的温度及组分分布 等信息, 从而指导实际燃烧装置的优化和改进。平 面激光诱导荧光技术是一种利用片状光束诊断燃烧 场物理信息的激光测量技术 ${ }^{[5]}$, 它利用激光片光整形 系统将激光器输出的圆光斑整形成线光斑, 进而探 测被测燃烧区的物理场。然而, 现有激光器输出的 高斯或类高斯光束能量分布都不均匀, 因而通过整
形系统后输出的线光斑能量分布也是不均匀的, 利 用这种光束测量燃烧场, 将导致探测信号的信噪比 出现不一致的情况（有些区域的信噪比高, 而有些 区域的信噪比很低), 极大地影响了激光燃烧诊断的 测量精度。

为消除高斯光束能量分布不均匀性给上述研究 领域带来的不良影响，人们发展了大量激光光束均 匀化方法, 如非球面透镜组整形法、衍射光学元件 法、随机相位法、液晶空间光调制法以及自聚焦透 镜法 ${ }^{[6-8]}$ 。龚华平等人系统总结了激光束空间整形的 研究现状, 比较了二元光学元件、光楔列阵聚焦光 学系统、液晶空间光调制器、双折射透镜组和随机 相位板这些光束整形方法之间的优缺点 ${ }^{[9]}$ 。表 1 给出 了现有主流光束均匀化方法之间的优点和缺陷。

\section{表 1 现有光束均匀化方法优缺点比较}

Table 1 Comparison of advantages and disadvantages for the current beam uniformization methods

\section{Beam shaping \\ method \\ Advantages \\ Disadvantages}

High diffraction effi- The plastic quality is ciency; adjustable restricted by the level spot profile; micro, of non-fine machining

Binary optics array, integration and technology, therefore arbitrary wavefront the laser damage thretransformation shold is low

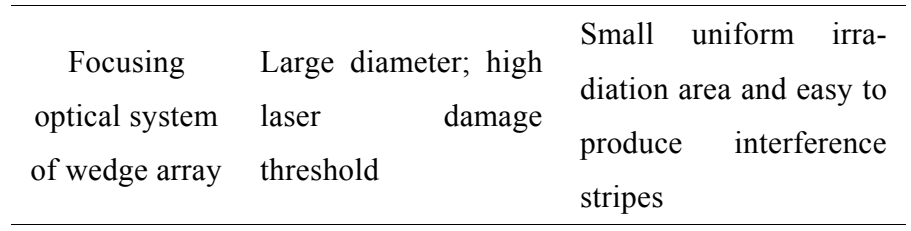

\begin{tabular}{cll}
$\begin{array}{c}\text { Liquid crystal } \\
\text { spatial light } \\
\text { modulator }\end{array}$ & $\begin{array}{l}\text { Real-time and adjust- } \\
\text { table spot shape }\end{array}$ & $\begin{array}{l}\text { Low laser damage } \\
\text { threshold }\end{array}$ \\
\hline $\begin{array}{c}\text { Birefringent } \\
\text { Lenses }\end{array}$ & $\begin{array}{l}\text { The transmissivity } \\
\text { function can be } \\
\text { changed flexibly }\end{array}$ & $\begin{array}{l}\text { High processing diffi- } \\
\text { culty }\end{array}$ \\
\hline
\end{tabular}
Easy to control the

Random phase shape and size of the Interference speckle on plate focal spot, simple to target make

\begin{tabular}{cl}
\hline \multirow{2}{*}{ Aspheric } & Easy processing and \\
lenses shaping high laser damage Low flexibility & \\
& threshold
\end{tabular}


通过表 1 可以看出, 采用现有光束均匀方法只 能将高斯光束在成像面处整形为能量分布均匀的光 斑, 在远离像面的位置处, 光束能量急剧发散 (或 会聚), 即整形后的光束仍存在很大发散角 (或会聚 角)。在强激光损伤阈值测量、激光清洗及激光燃烧 诊断领域中, 常常期望整形后的均匀平顶高斯光束 在一定距离范围内的发散角基本不发生变化, 而现 有高斯光束均匀化光学系统无法满足这一需求。

为此, 本文基于非球面镜的像差效应设计了一种 新型激光光束均匀化光学系统, 不仅能够实现对高斯 光束的均匀化, 还保证了距像面 $\pm 100 \mathrm{~mm}$ 处的光束 均匀性不发生大的改变, 在焦深范围内的光束均匀性 $\geqslant 95 \%$, 设计后的激光光束会聚角为 $17.52 \mathrm{mrad}$, 在 焦深范围内基本不改变光束直径。

\section{1 设计思想}

对于理想的光学系统而言, 光轴上任意一点发 出的光线通过光学系统的任一部分都可以在像面初 汇聚成一个完美的点。实际情况却非如此, 由于光 学系统中采用的镜片加工工艺和精度与理想情况存 在偏差, 在球面镜片组成的光学系统中, 由于在边 缘部分光线所发生的折射弯曲程度比中心要大, 因 此通过镜片边缘的光线会偏离汇聚点。大量光线通 过镜片边缘时, 在像面处就无法形成清晰的物像。 此即为球面像差, 又称球差。图 1 为理想光学系统 和实际光学系统的成像示意图。

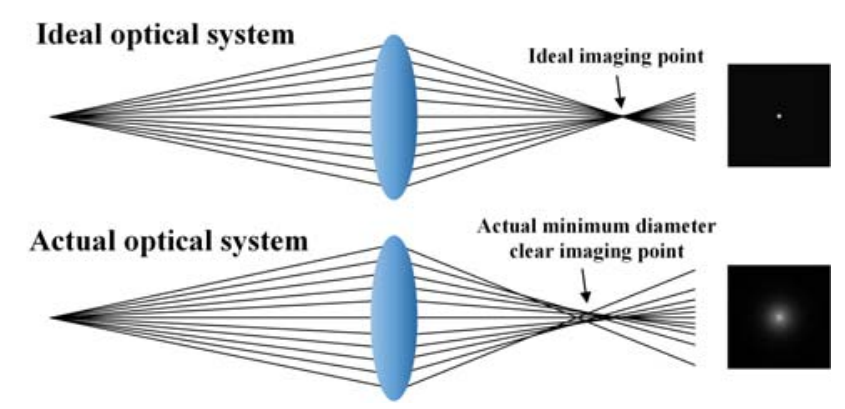

图 1 球面像差示意图

Fig.1 Schematic of spherical aberration

采用球面镜对实际物体成像, 由于总是存在球 面像差效应, 因此实际成像点往往是模糊的。在以 往的光学系统中, 往往期望获得清晰的物像, 因而 这些光学系统设计时往往需要对像差进行校正 ${ }^{[10]}$ 。 相反地, 我们看到球差效应也能够使得理想光学系 统中的理想清晰成像点变为一模糊发散的不清晰成 像点。若利用实际光学系统中的像差效应, 通过特 殊的非球面镜面面型设计, 将高斯光束中间能量集 中的部分投影到两侧能量较弱的位置, 就能够将高
斯光束转换成平顶高斯分布, 以达到光束均匀化的 目的。这一工作在此前研究中未见报道。

另外, 由于以往的光束能量均匀化方法仅能将 高斯光束在像面位置处转化为平顶高斯分布, 本文 为实现长焦深光束能量均匀化整形设计, 考虑在整 个光学系统中增加望远准直系统, 以达到长焦深的 设计目的。实际上, 长焦深和非球面镜像差本质上 是一个相互矛盾的关系, 非球面镜像差效应实际上 是将原本入射方向有序的光束转换为无序的光束, 而无序的光束通过透镜组的设计实际上是无法完全 实现真正意义上的准直的。因而本文设计的长焦深 光束均匀化光学系统具有有限焦深。

\section{2 性能指标}

基于非球面镜镜像差效应的激光光束均匀化光 学系统设计参数如表 2 所示。本文光学系统设计中, 为实现长焦深高斯光束均匀化整形, 降低实际非球 面镜片的加工难度, 应尽可能考虑满足如下要求:

1）为降低加工难度, 系统中应尽可能采取最少 的非球面镜面, 本文采用一个非球面镜面面型实现 将高斯光束向平顶高斯光束的整形变换;

2）尽可能保证焦深范围内光束均匀性不发生大 的变化, 本文设计的光束均匀度 $\geqslant 95 \%$, 能够满足实 际需求;

3) 为满足实际工作距离变化范围大的需求, 设 计的光束整形系统焦深尽可能大且光束均匀性不发 生大的变化，本文设计焦深为 $\pm 100 \mathrm{~mm}$;

4) 尽可能实现高光学传输效率, 本文设计时对 所有透镜镜面采用增镀增透膜, 可实现光学传输效 率 99.9\%。

表 2 光学系统性能参数

Table 2 Performance parameters of optical system

\begin{tabular}{lc}
\hline \multicolumn{1}{c}{ Performance parameter } & Design value \\
\hline Wavelength & $1.064 \mu \mathrm{m}$ \\
Gaussian beam diameter & $10 \mathrm{~mm}$ \\
Laser withstand power & $300 \mathrm{~W}$ \\
Beam uniformity & $\geqslant 95 \%$ \\
Transmission efficiency of optical & $\geqslant 99.9 \%$ \\
$\quad$ system & $1000 \mathrm{~mm}$ \\
System focal length & $\pm 100 \mathrm{~mm}$ \\
System focal depth & $0 \mathrm{mrad}$ \\
Laser divergence angle of entrance \\
$\quad$ pupil
\end{tabular}

为保证光学系统的光学系统传输效率达到 
99.9\%，本文设计时在每片镜片前后表面加镀了增透 膜。而为更好地将高斯光束中间能量较高部分投影 至边缘部分，从而保证高斯光束的均匀性能够达到 $95 \%$ 以上, 设计时引入了一个偶次非球面。整个光学 系统由 9 片透镜组成, 其中第 5 面为偶次非球面。 引入的偶次非球面, 其表达式 ${ }^{[11]}$ 如下式所示:

$$
z=\frac{c r^{2}}{1+\sqrt{1-(1+k) c^{2} r^{2}}}+a_{1} r^{2}+a_{2} r^{4}+a_{3} r^{6}+a_{4} r^{8}+\cdots
$$

式中: $c=1 / r_{0}$, 本文中 $r_{0}=-13.422 ; k=-\mathrm{e}^{2} ; a_{1}$ 、 $a_{2} 、 a_{3} 、 a_{4}$ 为高次项系数; $r$ 为归一化半径坐标; $c$ 为非球面的基准面或辅助球面的曲率; $k$ 为锥面度。 本文设计时, 为考虑降低实际加工难度, 只选取了 式(1)中的雉面度 $k$, 且 $k=-3.74$ 。其他高次球面系 数的设计值均为 0 。

基于非球面像差效应设计的长焦深高斯光束均 匀化光学系统中各镜片参数 (顺序为从左向右) 如 表 3 所示, 表中半径和厚度的单位为 $\mathrm{mm}$ 。

表 3 光学系统元素表 Table 3 Optical system elements table

\begin{tabular}{|c|c|c|c|c|c|}
\hline Lens & $\begin{array}{c}\text { Surface } \\
\text { shape }\end{array}$ & $\begin{array}{l}\text { Radius } \\
\text { /mm }\end{array}$ & $\begin{array}{c}\text { Thickness } \\
\text { /mm }\end{array}$ & $\begin{array}{c}\text { Quadric } \\
\text { surface } \\
\text { coefficient }\end{array}$ & $\begin{array}{l}\text { Higher } \\
\text { aspheric } \\
\text { coefficient }\end{array}$ \\
\hline \multirow{2}{*}{$1 \mathrm{st}$} & Spherical & 10.550 & \multirow{2}{*}{4.1} & 0 & \\
\hline & Spherical & 7.810 & & 0 & \\
\hline \multirow{2}{*}{ 2nd } & Spherical & -26.507 & \multirow{2}{*}{4.6} & 0 & \\
\hline & Spherical & -11.379 & & 0 & \\
\hline
\end{tabular}

\begin{tabular}{|c|c|c|c|c|c|}
\hline \multirow{2}{*}{$3 \mathrm{rd}$} & Aspheric & -13.422 & \multirow{2}{*}{3.1} & -3.74 & \\
\hline & Spherical & -32.824 & & 0 & \\
\hline \multirow{2}{*}{4 th } & Spherical & -51 & \multirow{2}{*}{3.2} & 0 & \\
\hline & Spherical & 19.7 & & 0 & 0 \\
\hline \multirow{2}{*}{5 th } & Spherical & -39.519 & \multirow{2}{*}{6.8} & 0 & \\
\hline & Spherical & -24.834 & & 0 & \\
\hline \multirow{2}{*}{6 th } & Spherical & 27.599 & \multirow{2}{*}{8.8} & 0 & \\
\hline & Spherical & 251.380 & & 0 & \\
\hline \multirow{2}{*}{7 th } & Spherical & -22.763 & \multirow{2}{*}{4.6} & & \\
\hline & Spherical & -170.08 & & 0 & \\
\hline \multirow{2}{*}{ 8th } & Spherical & -600.036 & \multirow{2}{*}{6.6} & 0 & \\
\hline & Spherical & -43.026 & & 0 & \\
\hline \multirow{2}{*}{ 9th } & Spherical & -30.851 & \multirow{2}{*}{5} & 0 & \\
\hline & Spherical & -41.139 & & 0 & \\
\hline
\end{tabular}

\section{3 设计结果与分析}

\section{1 设计结果}

通过 Zemax 软件进行序列分析和光线追迹, 优 化后得到的基于非球面像差效应的长焦深激光光束 均匀化系统的光学结构如图 2 所示。

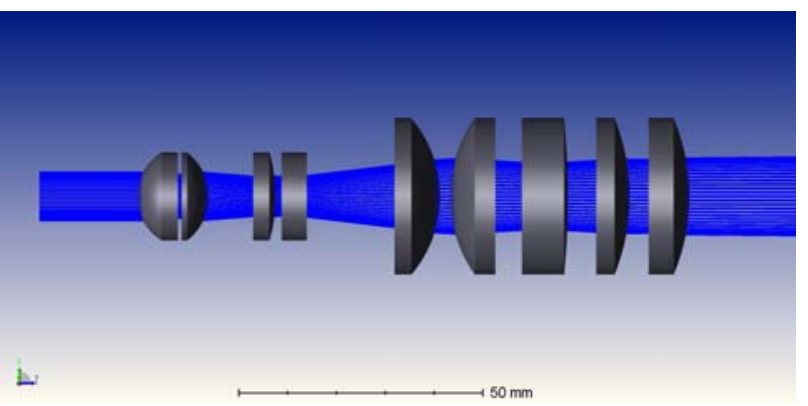

图 2 长焦深高斯光束均匀化光学系统

Fig.2 The optical system of Gaussian beams uniformization with long focal depth

基于非球面像差效应的长焦深高斯激光光束均 匀化光学系统总体结构由光束均匀化非球面镜组 （沿光束传播方向上的前 5 枚透镜）和长焦深球面准 直镜组组成（其余 4 枚透镜），其中光束自左向右传 播。光束均匀化非球面镜组的功能是将高斯光束中 间能量集中部分投影至两侧能量较弱的位置, 而长 焦深球面准直镜组的功能则是将已经均匀化的平顶 高斯光束进一步准直, 在 $1000 \mathrm{~mm} \pm 100 \mathrm{~mm}$ 处形成 能量分布均匀的光斑。其中焦距 $f=1000 \mathrm{~mm}$, 这一 距离为焦平面中心距离光学系统最后一片镜面中心 之间的距离。

设计后的光学系统结构参数为: 工作波段为 $1064 \mathrm{~nm}$, 耐受激光功率为 $300 \mathrm{~W}$, 有效焦距为 1000 $\mathrm{mm}$, 有效焦深为 $\pm 100 \mathrm{~mm}$, 光学系统传输效率为 $99.9 \%$, 系统总长为 $135.2 \mathrm{~mm}$ 。

\section{2 分析讨论}

从图 2 中可以看到, 当高斯光束经过本文设计 的光束均匀化光学系统时, 出瞳后的光斑直径要大 于入射光斑的直径。这是由于光束均匀化和长焦深 这两者互相矛盾所导致的。光束均匀化本质上是通 过非球面像差效应将原本发散角固定的入射光线集 合通过非球面镜面重新排列而实现的。光线经重新 排列后, 每一条子光线的传播方向虽在像面处能够 形成能量分布均匀的光斑, 但其与光轴之间的夹角 将不再一致而变得杂乱, 因而通过准直透镜组后各 光线的传播方向仍无法完全恢复，但可以将光束的 发散角在焦深范围内控制在可接受的程度。本文中, 在焦深范围内光束的会聚角为 $17.52 \mathrm{mrad}$, 能够满足 
实际需求。

图 3 给出了距焦平面距离为 $-200 \sim 200 \mathrm{~mm}$ 范 围内的光斑能量空间能量分布情况。从图 $3(\mathrm{a}) \sim(\mathrm{g})$ 中可看出, 焦深 $\pm 100 \mathrm{~mm}$ 范围内的光斑中心能量分 布均匀性很好, 原高斯光束中间集中的能量已被均 匀分配至两侧。

然而, 根据光学理论中的拉氏不变定理, 像高 与物高之比等于像方发散角与物方发散角正切之 比。因此在本文光学系统中, 经过整形后的平顶高 斯光束仍均有一定会聚角。由于整形光束会聚角的 存在, 在焦深范围内的光束非相干辐照度和光斑直 径都将有所不同。

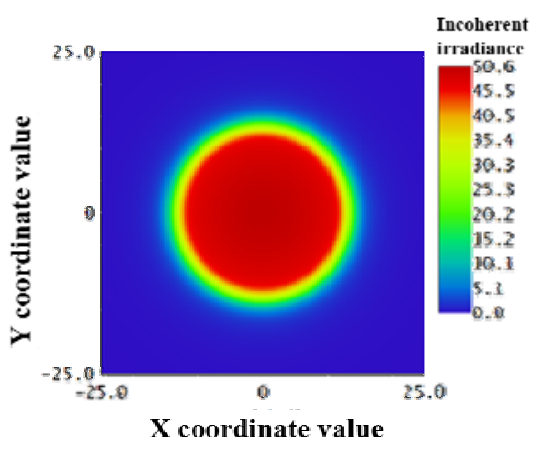

(a) 距焦平面 $-200 \mathrm{~mm}$ 处光斑能量分布

(a) Energy distribution at $-200 \mathrm{~mm}$ from focal plane

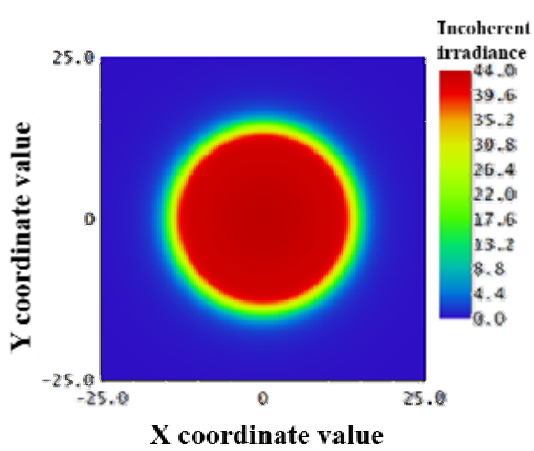

(b) 距焦平面 $-100 \mathrm{~mm}$ 处光斑能量分布

(b) Energy distribution at $-100 \mathrm{~mm}$ from focal plane

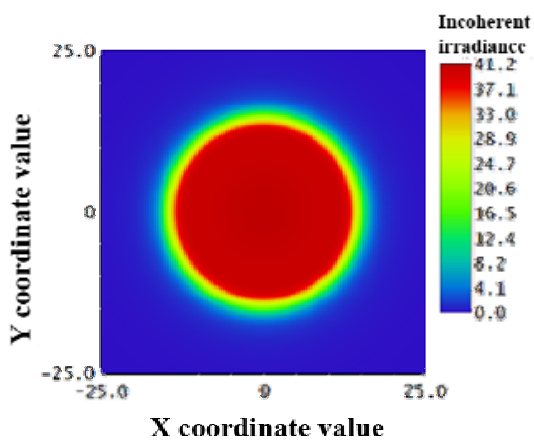

(c) 距焦平面 $-50 \mathrm{~mm}$ 处光斑能量分布

(c) Energy distribution at $-50 \mathrm{~mm}$ from focal plane

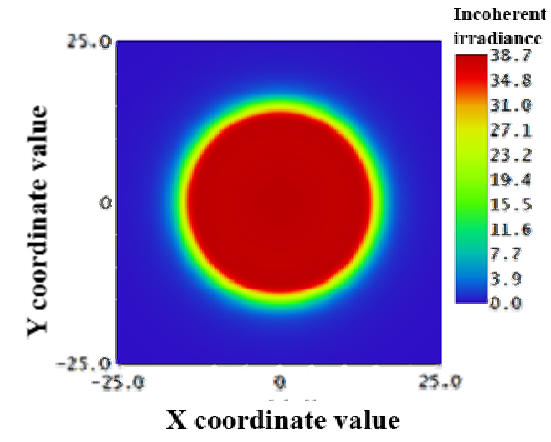

(d) 焦平面处光斑能量分布

(d) Energy distribution at focal plane

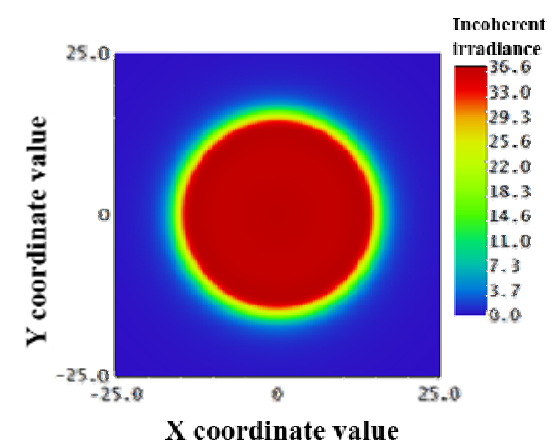

(e) 距焦平面 $50 \mathrm{~mm}$ 处光斑能量分布

(e) Energy distribution at $50 \mathrm{~mm}$ from focal plane

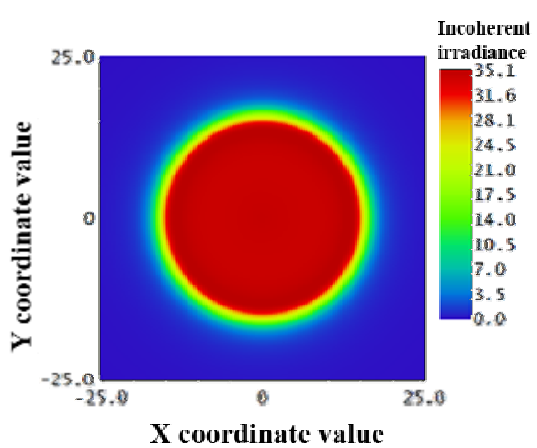

(f) 距焦平面 $100 \mathrm{~mm}$ 处光斑能量分布

(f) Energy distribution at $100 \mathrm{~mm}$ from focal plane

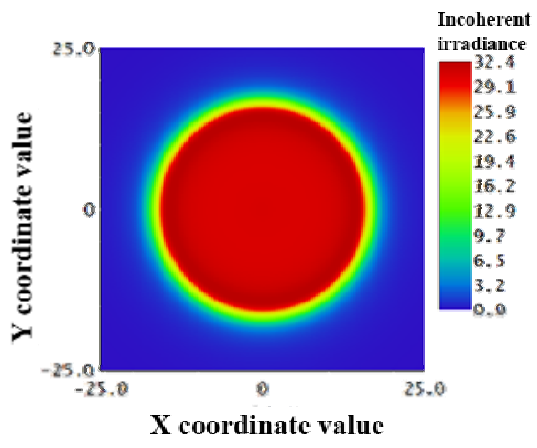

(g) 距焦平面 $200 \mathrm{~mm}$ 处光斑能量分布

(g) Energy distribution at $200 \mathrm{~mm}$ from focal plane 图 3 距焦平面不同位置处光斑能量分布

Fig. 3 Distribution of spot energy at different positions from focal plane 
图 4 给出了距离焦平面 $-200 \sim 200 \mathrm{~mm}$ 范围内 各典型位置的光斑中心行截面非相干辐照度分布情 况。图 4 中 “一”号表示与光束传播方向相反, “+” 号表示与光束传播方向相同。从图 4 中可以看出, 光束的非相干辐照度随着距焦平面的距离增大而降 低, 宽度也随之增大。

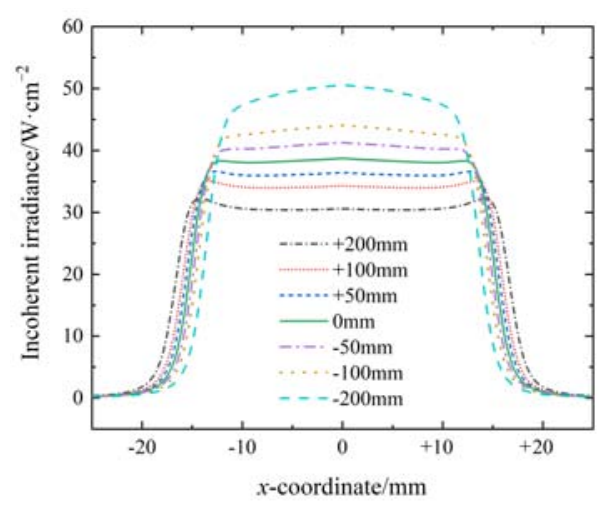

图 4 光斑中心行截面辐照度 $(-200 \sim 200 \mathrm{~mm}$ )

Fig.4 The spot irradiance of the central row section $(-200-200 \mathrm{~mm})$

图 5 给出了距离焦平面不同距离处的光斑非相 干辐照度最大值分布情况。可以看出, 当距焦平面 位置从-200 $\mathrm{mm}$ 依次增大到 $200 \mathrm{~mm}$ 时, 整形后光 斑能量最大值也随之逐渐增大, 且其变化规律与抛 物线二次拟合函数相符合，拟合后的二次多项式函 数表达式为: $y=6.7116 \times 10^{-5} x^{2}+0.04546 x+$ 38.79113。

通过上述分析, 可以看出, 光束在传播过程中, 由于存在会聚角而使得光斑的面积和能量都不是一 固定值, 而实际应用中则期望这种变化不大, 因此 实际焦深范围内的光束能量和光斑直径变化也应控 制在可接受的范围内。本文进一步考察了距离焦平 面不同位置处光束的非相干辐照度相对于焦平面处 的能量变化量, 其分布规律如图 6 所示。

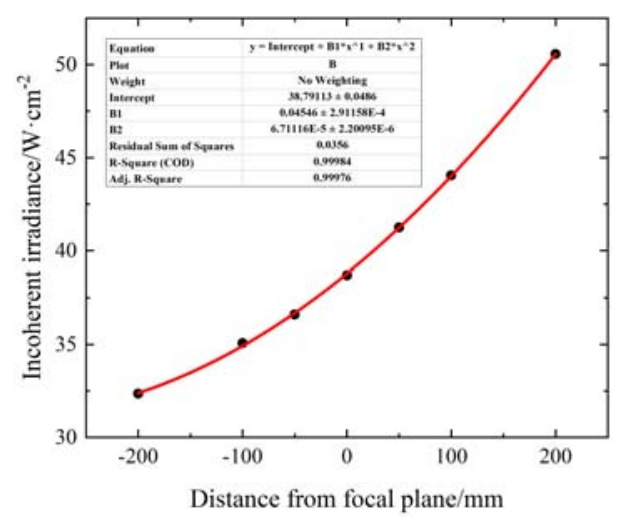

图 5 距焦平面不同位置处的光束能量最大值分布

Fig.5 Distribution of maximum beam energy at different positions of focal plane

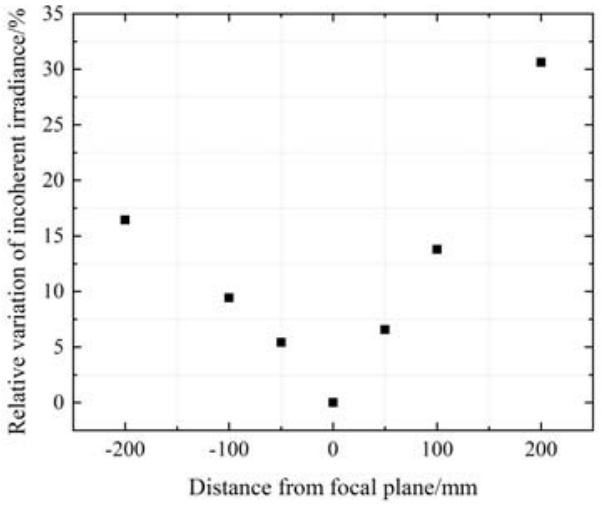

图 6 距焦平面不同位置处的非相干辐照度相对变化量

Fig.6 Relative variations of incoherent irradiance at different positions from focal plane

从图 6 中可以看出, 位于 $-200 \mathrm{~mm}$ 和 $200 \mathrm{~mm}$ 处的光束非相干辐照度相对于焦平面处的变化量分 别为 $16.4 \%$ 和 $30.6 \%$, 在实际工程应用和研究中难以 应用。而位于 $-100 \mathrm{~mm}$ 和 $100 \mathrm{~mm}$ 处的非相干辐照 度变化量最大为 $13.7 \%$, 实际应用时在可接受范围。 因此, 本文设计的长焦深高斯光束均匀化光学系统 的焦深为 $\pm 100 \mathrm{~mm}$, 且在这一范围内光束的能量变 化量不大于 $13.7 \%$ 。

光束均匀性反映了均光系统对标准高斯光束能 量分布的均化能力, 是一项重要的技术指标。为此, 本文计算了不同焦平面处的光束均匀度, 其结果如 图 7 所示。本文对平顶高斯光束均匀性作如下定义:

$$
\eta=1-\operatorname{Max}\left|\frac{E_{i}-\bar{E}}{\bar{E}}\right|
$$

式中: $\eta$ 为光束均匀度; $E_{i}$ 为平顶高斯光束宽度内各 点的非相干辐照度; $\bar{E}$ 为平顶高斯光束宽度内的平均 非相干辐照度; “Max” 表示对各点计算结果取最大 值。

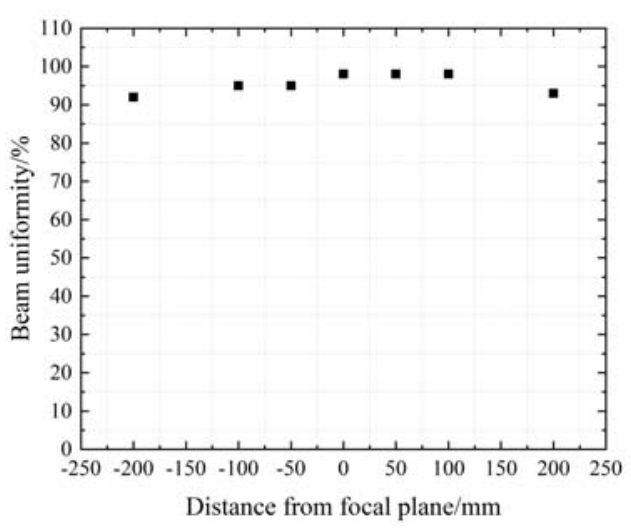

图 7 距焦平面不同位置处的光束均匀度分布

Fig.7 Distribution of beam uniformity at different positions from focal plane 
可以看到, 在距离焦平面 $\pm 200 \mathrm{~mm}$ 范围内的各 典型位置处, 光束均匀度最小为 $92 \%$, 最大为 $98 \%$ 。 而在焦深范围内 $( \pm 100 \mathrm{~mm})$, 光束均匀度 $\geqslant 95 \%$, 这一均匀性是能够满足绝大多数应用场景的。

光斑宽度和平均非相干辐照度也是实际应用中 关心的技术参数, 为此考察了不同焦平面距离处的光 斑宽度和平均辐照度分布情况, 其结果如图 8 所示。

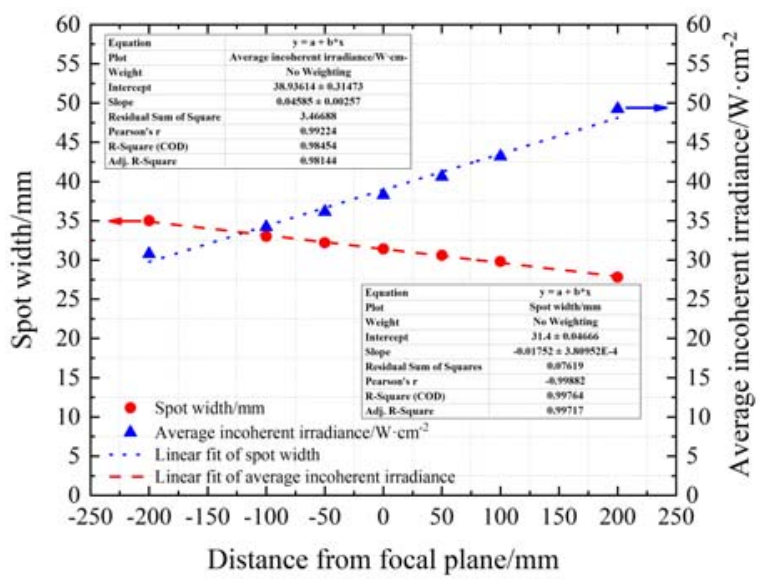

图 8 距焦平面不同位置处光斑宽度和平均辐照度分布

Fig. 8 Distributions of the spot width and the mean incoherent irradiance at different positions from focal plane

可以看出, 光斑宽度随距离的增加而线性减小, 而光束平均非相干辐照度则随距离增加线性增加。 线性拟合后的光斑宽度和光束非相干辐照度表达式 分别为: $y=-0.01752 x+31.4$ 和 $y=0.04585 x+38.9$ 。 利用上述光斑宽度的拟合关系, 计算出整形后的光 束在焦深范围内的会聚角为 $17.52 \mathrm{mrad}$ 。

\section{3 公差分析结果}

本文设计的长焦深高斯光束均匀化光学系统中 各镜片的加工要求如表 4 所示。其中 TTHI（total thickness) 表示镜片厚度公差 (单位为 $\mathrm{mm}$ ); $C$ 为偏 心度 (单位为 $\mathrm{mm}$ ); PV (peak value) 为干涉仪测得 的波前最高点和最低点的差值 (单位为波长 $\lambda$ ); RMS (root mean square) 为均方根粗糙度 (单位为波长 $\lambda$ )。

采用 10000 次蒙特卡洛计算, 结果表明弥散斑 半径变化量最大值为 $424 \mu \mathrm{m}$, 小于 $240 \mu \mathrm{m}$ 的概率为 $98 \%$, 此时后截距补偿范围为 $\pm 17 \mathrm{~mm}$ 。本文各光学 镜片 (即从第 1 片透镜到第 9 片透镜) 的安装偏差 要求均为: 位置偏心最大 $0.1 \mathrm{~mm}$, 位置倾角不超过 $0.05^{\circ}$ 。

对于应用于高能激光作用下的高精度非球面加 工, 目前常采用传统的研磨抛光工艺, 利用抛光盘 和工件表面的相对滑动, 借助抛光液和混合其中的 磨料粒子与工件表面间的机械化学和物理作用, 加
工该非球面达到接近球面，后续采用机修和手修相 结合的方法实现非球面的加工。加工完成后制作该 非球面镜的补偿镜, 两者结合输出球面波使用干涉 仪可以检测出非球面的 PV 和 RMS 值。

表 4 各光学镜片加工要求

Table 4 Processing requirements for optical lens

\begin{tabular}{|c|c|}
\hline Sequence number of lens & Deviation \\
\hline \multirow{4}{*}{1 st } & $\mathrm{TTHI}=0.02 \mathrm{~mm}$ \\
\hline & $C=0.05 \mathrm{~mm}$ \\
\hline & $\mathrm{PV} \leq 0.25 \lambda$ \\
\hline & $\mathrm{RMS} \leq 0.05 \lambda$ \\
\hline \multirow{4}{*}{ 2nd } & $\mathrm{TTHI}=0.03 \mathrm{~mm}$ \\
\hline & $\mathrm{C}=0.05 \mathrm{~mm}$ \\
\hline & $\mathrm{PV} \leq 0.25 \lambda$ \\
\hline & $\mathrm{RMS} \leq 0.05 \lambda$ \\
\hline \multirow{4}{*}{$3 \mathrm{rd}$} & $\mathrm{TTHI}=0.03 \mathrm{~mm}$ \\
\hline & $C=0.05 \mathrm{~mm}$ \\
\hline & $\mathrm{PV} \leq 0.25 \lambda$ \\
\hline & $\mathrm{RMS} \leq 0.05 \lambda$ \\
\hline \multirow{4}{*}{4 th } & $\mathrm{TTHI}=0.03 \mathrm{~mm}$ \\
\hline & $C=0.05 \mathrm{~mm}$ \\
\hline & $\mathrm{PV} \leq 0.25 \lambda$ \\
\hline & $\mathrm{RMS} \leq 0.05 \lambda$ \\
\hline \multirow{4}{*}{5 th } & $\mathrm{TTHI}=0.02 \mathrm{~mm}$ \\
\hline & $C=0.05 \mathrm{~mm}$ \\
\hline & $\mathrm{PV} \leq 0.25 \lambda$ \\
\hline & $\mathrm{RMS} \leq 0.05 \lambda$ \\
\hline \multirow{4}{*}{6 th } & $\mathrm{TTHI}=0.03 \mathrm{~mm}$ \\
\hline & $C=0.05 \mathrm{~mm}$ \\
\hline & $\mathrm{PV} \leq 0.25 \lambda$ \\
\hline & $\mathrm{RMS} \leq 0.05 \lambda$ \\
\hline \multirow{4}{*}{7 th } & $\mathrm{TTHI}=0.03 \mathrm{~mm}$ \\
\hline & $C=0.05 \mathrm{~mm}$ \\
\hline & $\mathrm{PV} \leq 0.25 \lambda$ \\
\hline & $\mathrm{RMS} \leq 0.05 \lambda$ \\
\hline \multirow{4}{*}{8 th } & $\mathrm{TTHI}=0.03 \mathrm{~mm}$ \\
\hline & $C=0.05 \mathrm{~mm}$ \\
\hline & $\mathrm{PV} \leq 0.25 \lambda$ \\
\hline & $\mathrm{RMS} \leq 0.05 \lambda$ \\
\hline \multirow{4}{*}{ 9th } & $\mathrm{TTHI}=0.03 \mathrm{~mm}$ \\
\hline & $C=0.05 \mathrm{~mm}$ \\
\hline & $\mathrm{PV} \leq 0.25 \lambda$ \\
\hline & $\mathrm{RMS} \leq 0.05 \lambda$ \\
\hline
\end{tabular}


综上所述, 本文基于非球面像差效应设计的长 焦深高斯光束均匀化光学系统在焦深 $\pm 100 \mathrm{~mm}$ 范围 内对高斯激光束的整形均匀度 $\geqslant 95 \%$, 会聚角为 $17.52 \mathrm{mrad}$, 最大非相干辐照度变化量为 $13.7 \%$, 该 系统具有光束均匀性高、发散角小、系统光学传输 效率高的优点, 能够满足激光清洗、激光损伤阈值 测量等实际应用。

\section{4 结论}

针对现有光束均匀化光学系统无法同时实现高 损伤激光功率和长焦深的设计, 本文基于非球面镜 像差效应设计了一种新型长焦深高斯激光光束均匀 化光学系统, 设计的光学整形系统同时实现了大焦 深和高斯光束能量空间分布的均匀化, 工作距离 $1000 \mathrm{~mm}$, 在焦深范围内的光束均匀性 $\geqslant 95 \%$, 会聚 角 $17.52 \mathrm{mrad}$, 耐受激光功率不小于 $300 \mathrm{~W}$ 。本文设 计的长焦深高斯激光束均匀化光学系统能够为强激 光损伤阈值测量、激光清洗及激光燃烧诊断领域及 其他相关技术领域提供重要的技术支撑，具有广阔 的应用前景。

\section{参考文献:}

[1] 黄亿辉, 袁武, 宋宏伟, 等. CW 激光辐照薄板热力响应全场测量 [J]. 强激光与粒子束, 2014, 26(11): 111012 .

HUANG Yihui, YUAN Wu, SONG Hongwei, et al. Full-field measurement of thin plate irradiated by $\mathrm{CW}$ laser[J]. High Power Laser and Particle Beams, 2014, 26(11): 111012.

[2] 叶亚云. 光学元件表面的激光清洗技术研究[D]. 绵阳: 中国工程物 理研究院, 2010.

YE Yayun. Research on Laser Cleaning Technology for Optical Component Surface[D]. Mianyang: China Academy Of Engineering Physics, 2010.

[3] 胡志云, 刘晶儒, 张振荣, 等. 激光燃烧诊断技术及应用研究进展 [J]. 中国工程科学, 2009, 11(11): 45-50.

HU Zhiyun, LIU Jingru, ZHANG Zhenrong, et al. The research progress of laser combustiondiagnostics techniques and applications[J]. Engineering Science, 2009, 11(11): 45-50.
[4] 雷正龙, 田泽, 陈彦宾. 工业领域的激光清洗技术 $[\mathrm{J}]$. 激光与光电 子学进展, 2018, 55(3): 030005.

LEI Zhenglong, TIAN Ze, CHEN Yanbin. Laser cleaning technology in industrial fields[J]. Laser \& Optoelectronics Progress, 2018, 55(3): 030005 .

[5] 陈爽, Lukasz Jan Kapusta, 翁武斌, 等. $\mathrm{CH}_{4} / \mathrm{Air}$ 反扩散射流火焰多 组分同步 PLIF 诊断[J]. 实验流体力学, 2018, 32(1): 26-32.

CHEN Shuang, Lukasz Jan Kapusta, WENG Wubin, et al. Simultaneous multi-species PLIF diagnostic on $\mathrm{CH}_{4}$-air inverse diffusion jet flame[J]. Journal of Experiments in Fluid Mechanics, 2018, 32(1): 26-32.

[6] 林勇, 胡家升. 激光光束的整形技术 [J]. 激光杂志, 2008, 29(6): 1-4. LIN Yong, HU Jiasheng. Laser beam shaping techniques[J]. Laser Journal, 2008, 29(6): 1-4.

[7] Dickey F M, Holswade S C. Laser Beam Shaping: Theory and Techniques[M]. New York: Marcel Dekker Inc., 2000.

[8] 陈凯. 高斯光束整形为平顶光束整形系统的研究与设计 [D]. 北京: 北京工业大学, 2011.

CHEN Kai. The Research and Design of the System that Converting a Gaussian to a Flattop Beam[D]. Beijing: Beijing University of Technology, 2011.

[9] 枈华平, 吕志伟, 林殿阳. 激光束空间整形的研究现状[J]. 激光与 光电子学进展, 2005, 42(9): 2-5.

GONG Huaping, LYU Zhiwei, LIN Dianyang. Present status of laser beam spatial shaping[J]. Laser \& Optoelectronics Progress, 2005, 42(9): 2-5.

[10] 付薇, 陈宝国. 基于非球面固定校正镜的共形光学系统设计 [J]. 红 外技术, 2010, 32(7): 408-410.

FU Wei, CHEN Baoguo. Design of a conformal optical system based on fixed aspheric corrector[J]. Infrared Technology, 2010, 32(7): 408-410.

[11] 陈潇. 大面阵长波红外光学无热化镜头的设计 [J]. 红外技术, 2018 , 40(11): 1061-1064.

CHEN Xiao. Design of long-wavelength infrared athermalization lens for large-array detector[J]. Infrared Technology, 2018, 40(11): 1061-1064. 